\title{
High-Order Central WENO Schemes for 1D Hamilton-Jacobi Equations
}

\author{
Steve Bryson ${ }^{1}$ and Doron Levy ${ }^{2}$ \\ 1 Program in Scientific Computing/Computational Mathematics, Stanford \\ University and the NASA Advanced Supercomputing Division, NASA Ames \\ Research Center, Moffett Field, CA 94035-1000; bryson@nas.nasa.gov \\ 2 Department of Mathematics, Stanford University, Stanford, CA 94305-2125; \\ dlevy@math.stanford.edu

\section{Introduction}

We consider high-order central approximations for solutions of one-dimensional Hamilton-Jacobi (HJ) equations of the form

$$
\frac{\partial}{\partial t} \phi(x, t)+H\left(\phi_{x}, x\right)=0, \quad x \in \mathbb{R}
$$

subject to the initial data $\phi(x, t=0)=\phi_{0}(\mathbf{x})$. Solutions for (1) with smooth initial data typically remain continuous but develop discontinuous derivatives in finite time. Such solutions are not unique; the physically relevant solution is known as the viscosity solution (see $[1,3,4,5,8,15]$ and the references therein).

Various numerical methods were proposed in order to approximate the solutions of (1). Examples for such methods are the high-order Godunov-type schemes that were introduced in $[20,21]$, and were based on an Essentially Non-Oscillatory (ENO) reconstruction step [7] that was evolved in time with a first-order monotone flux. The least dissipative monotone flux, the Godunov flux, requires solving Riemann problems at cell interfaces. A fifth-order Weighted ENO (WENO) scheme, based on $[10,18]$, was introduced by Jiang and Peng [9].

Recently, Lin and Tadmor introduced in $[16,17]$ central schemes for approximating solutions of the HJ equation. These schemes are based on the Nessyahu-Tadmor scheme for approximating solutions of hyperbolic conservation laws [19]. Unlike upwind schemes, central schemes do not require Riemann solvers, which makes them attractive for solving systems of equations and for multi-dimensional problems. A second-order semi-discrete version of these schemes was introduced by Kurganov and Tadmor in [12]. While less dissipative, the semi-discrete scheme requires the estimation of the local speed of propagation, which is computationally intensive in particular in multi-dimensional problems. In a later work [11], the numerical viscosity was further reduced by computing more precise information about local speed of propagation. To address the problem of schemes that are too computationally intensive, we introduced in [2] efficient first- and second-order central schemes for approximating the solutions of multi-dimensional versions of (1). 
Unlike the previous attempts, our schemes in [2] scale well with increasing dimension.

In this paper we derive fully-discrete Central WENO (CWENO) schemes for approximating solutions of (1), which combine our previous works $[2$, $13,14]$. We introduce third- and fifth-order accurate schemes, which are the first central schemes for the HJ equations of order higher than two. The core ingredient in the derivation of our schemes is a high-order CWENO reconstructions in space.

Acknowledgment: We would like to thank Volker Elling for helpful discussions.

\section{CWENO Schemes for HJ Equations}

We are interested in approximating solutions of (1) subject to the initial data $\phi(x, t=0)=\phi_{0}(x)$. For simplicity we assume a uniform grid grid in space and time with mesh spacings, $h:=\Delta x$ and $\Delta t$. We denote the grid points by $x_{i}=i \Delta x, t^{n}=n \Delta t$, and the fixed mesh ratio by $\lambda=\Delta t / \Delta x$. Let $\varphi_{i}^{n}$ denote the approximate value of $\phi\left(x_{i}, t^{n}\right)$, and $\left(\varphi_{x}\right)_{i}^{n}$ denote the approximate value of the derivative $\phi_{x}\left(x_{i}, t^{n}\right)$. We define $\Delta^{+} \varphi_{i}^{n}:=\varphi_{i+1}^{n}-\varphi_{i}^{n}, \Delta^{-} \varphi_{i}^{n}:=\varphi_{i}^{n}-\varphi_{i-1}^{n}$ and $\Delta^{0} \varphi_{i}^{n}:=\varphi_{i+1}^{n}-\varphi_{i-1}^{n}$.

We assume that the approximate solution at time $t^{n}, \varphi_{i}^{n}$ is given. In order to approximate the solution at the next time step $t^{n+1}, \varphi_{i}^{n+1}$, we start by reconstructing a continuous piecewise-polynomial from the data, $\varphi_{i}^{n}$, and sample it at the half-integer points, $\left\{x_{i+1 / 2}\right\}$, in order to obtain the pointvalues of the interpolant at these points $\varphi_{i+1 / 2}^{n}$ as well as the derivative, $\varphi_{i+1 / 2}^{\prime}$. We then evolve $\varphi_{i+\frac{1}{2}}^{n}$ from time $t^{n}$ to time $t^{n+1}$ according to (1),

$$
\varphi\left(x_{i+\frac{1}{2}}, t^{n+1}\right)=\varphi\left(x_{i+\frac{1}{2}}, t^{n}\right)-\int_{t^{n}}^{t^{n+1}} H\left(\varphi_{x}\left(x_{i+\frac{1}{2}}, t\right)\right) d t .
$$

This evolution is done at the half-integer grid points where the reconstruction is smooth (as long as the CFL condition $\lambda\left|H^{\prime}\left(\varphi_{x}\right)\right| \leq 1 / 2$ is satisfied). Finally, in order to return to the original grid, we project $\varphi_{i+1 / 2}^{n+1}$ back onto the integer grid points $\left\{x_{i}\right\}$ to end up with $\varphi_{i}^{n+1}$.

Since the evolution step (2) is done at points where the solution is smooth, we can approximate the time integral at the RHS of (2) using a sufficiently accurate quadrature rule. For example, for a third- and fourth-order method, this integral can be replaced by a Simpson's quadrature,

$$
\begin{aligned}
\int_{t^{n}}^{t^{n+1}} H & \left(\varphi_{x}\left(x_{i+\frac{1}{2}}, t\right)\right) d t \approx \frac{\Delta t}{6}\left[H\left(\varphi_{x}\left(x_{i+\frac{1}{2}}, t^{n}\right)\right)\right. \\
& \left.+4 H\left(\varphi_{x}\left(x_{i+\frac{1}{2}}, t^{n+\frac{1}{2}}\right)\right)+H\left(\varphi_{x}\left(x_{i+\frac{1}{2}}, t^{n+1}\right)\right)\right] .
\end{aligned}
$$


The intermediate values of the derivative in time, $\varphi_{x}\left(x_{i+1 / 2}, t^{n+1 / 2}\right)$, and $\varphi_{x}\left(x_{i+1 / 2}, t^{n+1}\right)$, which are required in the quadrature (3), can be predicted using a Taylor expansion or with a Runge-Kutta (RK) method. For details we refer the reader to $[13,19]$ and the references therein.

The remaining ingredient is the piecewise-polynomial reconstruction in space. A careful study of the above procedure reveals that there are actually three different quantities that should be recovered in every time step. First, given $\varphi_{i}$ at time $t^{n}$ we need to reconstruct the point-values at the half-integer grid points, $\varphi_{i+1 / 2}$, at the same time $t^{n}$. This is the first term on the RHS of (2). The second term on the RHS of (2) requires evaluating the Hamiltonian $H$ at the derivative $\varphi_{i+1 / 2}^{\prime}$. Hence, the second quantity we should recover is $\varphi_{i+1 / 2}^{\prime}$ from $\varphi_{i}$. Finally, the predictor step that provides the values at the quadrature nodes in (3), require us to estimate $\varphi_{i+1 / 2}^{\prime}$ from $\varphi_{i+1 / 2}$ at every step of the RK method. In the next two sections we will focus on the reconstruction of these three quantities, first for a third-order method and then for a fifth-order method.

The projection from $\varphi_{i+1 / 2}^{n+1}$ onto the original grid points to get $\varphi_{i}^{n+1}$ is accomplished using the same reconstruction used to approximate $\varphi_{i+1 / 2}^{n}$ from $\varphi_{i}^{n}$.

\subsection{A Third-Order Scheme}

Following the above procedure, a third-order scheme can be generated by combining a third-order accurate ODE solver in time with a sufficiently high-order reconstruction in space. Here we present fourth-order CWENO reconstructions of the point values of $\varphi_{i+1 / 2}$ and its derivative $\varphi_{i+1 / 2}^{\prime}$.

The reconstruction of $\varphi_{i+1 / 2}$ from $\varphi_{i}$.

In order to obtain a fourth-order reconstruction of $\varphi_{i+1 / 2}$ we will write a convex combination of two quadratic polynomials, $\varphi_{-}^{[2]}$ constructed on a stencil which is left-biased with respect to $x_{i+1 / 2}$, and the right-biased $\varphi_{+}^{[2]}$,

$\varphi_{-}^{[2]}(x)=\varphi_{i}+\frac{1}{h}\left(\Delta^{+} \varphi_{i}\right)\left(x-x_{i}\right)+\frac{1}{2 h^{2}}\left(\Delta^{+} \Delta^{-} \varphi_{i}\right)\left(x-x_{i}\right)\left(x-x_{i+1}\right)+O\left(h^{3}\right)$, $\varphi_{+}^{[2]}(x)=\varphi_{i}+\frac{1}{h}\left(\Delta^{+} \varphi_{i}\right)\left(x-x_{i}\right)+\frac{1}{2 h^{2}}\left(\Delta^{+} \Delta^{+} \varphi_{i}\right)\left(x-x_{i}\right)\left(x-x_{i+1}\right)+O\left(h^{3}\right)$.

An evaluation of these approximations at $\left\{x_{i+\frac{1}{2}}\right\}$ reads

$\varphi_{-}^{[2]}\left(x_{i+\frac{1}{2}}\right)=\frac{1}{8}\left(-\varphi_{i-1}+6 \varphi_{i}+3 \varphi_{i+1}\right), \quad \varphi_{+}^{[2]}\left(x_{i+\frac{1}{2}}\right)=\frac{1}{8}\left(3 \varphi_{i}+6 \varphi_{i+1}-\varphi_{i+2}\right)$.

A straightforward computation shows that

$$
\frac{1}{2} \varphi_{-}^{[2]}\left(x_{i \frac{1}{2}}\right)+\frac{1}{2} \varphi_{+}^{[2]}\left(x_{i+\frac{1}{2}}\right)=\varphi_{i+\frac{1}{2}}+O\left(h^{4}\right) .
$$


The fourth-order WENO estimate of $\varphi_{i+1 / 2}$ is therefore given by the convex combination

$$
\varphi_{w}^{[4]}\left(x_{i+\frac{1}{2}}\right)=w_{i+\frac{1}{2}}^{-} \varphi_{-}^{[2]}\left(x_{i+\frac{1}{2}}\right)+w_{i+\frac{1}{2}}^{+} \varphi_{+}^{[2]}\left(x_{i+\frac{1}{2}}\right),
$$

where the weights satisfy $w_{i+1 / 2}^{-}+w_{i+1 / 2}^{+}=1, w_{i+1 / 2}^{ \pm} \geq 0, \forall i$. In smooth regions we would like to satisfy $w_{i}^{-} \approx w_{i}^{+} \approx \frac{1}{2}$ to attain an $O\left(h^{4}\right)$ error, while when the stencil $\left\{x_{i-1}, x_{i}, x_{i+1}, x_{i+2}\right\}$ supporting $\varphi_{w}\left(x_{i+\frac{1}{2}}\right)$ contains a discontinuity, the weight of the more oscillatory polynomial should vanish. Following $[10,18]$, we meet these requirements by setting

$$
w_{i+\frac{1}{2}}^{k}=\frac{\alpha_{i+\frac{1}{2}}^{k}}{\sum_{l} \alpha_{i+\frac{1}{2}}^{l}}, \quad \alpha_{i+\frac{1}{2}}^{k}=\frac{c^{k}}{\left(\epsilon+S_{i+\frac{1}{2}}^{k}\right)^{p}}
$$

where $k, l \in\{+,-\}$ ( $k$ and $l$ will range over a larger space of symbols when we use more interpolants). The constants $c^{ \pm}=1 / 2$ and are independent of the grid-point. We choose $\epsilon$ as $10^{-6}$ to prevents the denominator in (4) from vanishing, and set $p=2$ (see [10]). The smoothness measures $S_{i}^{ \pm}$should be large when $\varphi$ is nearly singular. Following the standard practice with WENOtype schemes [10], we take $S_{i}^{ \pm}$to be the sum of the $L^{2}$-norms of the first and second derivatives on the stencil supporting $\varphi_{ \pm}^{[2]}$. If we approximate the first derivative at $x_{i+1 / 2}$ by $\frac{1}{h} \Delta^{+} \varphi_{i+1 / 2}$, the second derivative by $\frac{1}{h^{2}} \Delta^{+} \Delta^{-} \varphi_{i+1 / 2}$, and define the smoothness measure

$$
S_{i+\frac{1}{2}}[r, s]=h \sum_{j=r}^{s}\left(\frac{1}{h} \Delta^{+} \varphi_{i+j+\frac{1}{2}}\right)^{2}+h \sum_{j=r+1}^{s}\left(\frac{1}{h^{2}} \Delta^{+} \Delta^{-} \varphi_{i+j+\frac{1}{2}}\right)^{2},
$$

then for the fourth-order interpolation of $\varphi_{w}\left(x_{i+\frac{1}{2}}\right)$ we have $S_{i+1 / 2}^{-}=$ $S_{i+1 / 2}[-1,0]$ and $S_{i+1 / 2}^{+}=S_{i+1 / 2}[0,1]$.

The reconstruction of $\varphi_{i+1 / 2}^{\prime}$ from $\varphi_{i}$.

To obtain a fourth-order estimate of the derivative $\varphi^{\prime}\left(x_{i+1 / 2}\right)$ from $\varphi\left(x_{i}\right)$, we start from the cubic interpolants

$$
\begin{aligned}
\varphi_{-}^{[3]}(x)= & \varphi_{i}+\frac{1}{h}\left(\Delta^{+} \varphi_{i}\right)\left(x-x_{i}\right)+\frac{1}{2 h^{2}}\left(\Delta^{+} \Delta^{-} \varphi_{i}\right)\left(x-x_{i}\right)\left(x-x_{i+1}\right) \\
& +\frac{1}{6 h^{3}}\left(\Delta^{-} \Delta^{+} \Delta^{-} \varphi_{i}\right)\left(x-x_{i}\right)\left(x-x_{i+1}\right)\left(x-x_{i-1}\right)+O\left(h^{4}\right) \\
\varphi_{+}^{[3]}(x)= & \varphi_{i}+\frac{1}{h}\left(\Delta^{+} \varphi_{i}\right)\left(x-x_{i}\right)+\frac{1}{2 h^{2}}\left(\Delta^{+} \Delta^{+} \varphi_{i}\right)\left(x-x_{i}\right)\left(x-x_{i+1}\right) \\
& +\frac{1}{6 h^{3}}\left(\Delta^{+} \Delta^{+} \Delta^{+} \varphi_{i}\right)\left(x-x_{i}\right)\left(x-x_{i+1}\right)\left(x-x_{i+2}\right)+O\left(h^{4}\right) .
\end{aligned}
$$

Differentiating $\varphi_{ \pm}^{[3]}$ at $x_{i+\frac{1}{2}}$ 


$$
\begin{aligned}
& \varphi_{-, i+\frac{1}{2}}^{\prime[3]}=\frac{1}{24 h}\left(\varphi_{i-2}-3 \varphi_{i-1}-21 \varphi_{i}+23 \varphi_{i+1}\right), \\
& \varphi_{+, i+\frac{1}{2}}^{\prime[3]}=\frac{1}{24 h}\left(-23 \varphi_{i}+21 \varphi_{i+1}+3 \varphi_{i+2}-\varphi_{i+3}\right) .
\end{aligned}
$$

Again,

$$
\frac{1}{2} \varphi_{-, i+\frac{1}{2}}^{\prime 3]}+\frac{1}{2} \varphi_{+, i+\frac{1}{2}}^{[3]}=\varphi_{i+\frac{1}{2}}^{\prime}+O\left(h^{4}\right)
$$

and a fourth-order WENO reconstruction of $\varphi^{\prime}\left(x_{i+\frac{1}{2}}\right)$ is

$$
\varphi_{i+1 / 2}^{[4]}=w_{i+\frac{1}{2}}^{-} \varphi_{-, i+\frac{1}{2}}^{\prime 33]}+w_{i+\frac{1}{2}}^{+} \varphi_{+, i+\frac{1}{2}}^{[33]}
$$

where the weights are of the form (4) with $c^{ \pm}=1 / 2$ and $S_{i+1 / 2}^{-}=$ $S_{i+1 / 2}[-2,0]$ and $S_{i+1 / 2}^{+}=S_{i+1 / 2}[0,2]$.

The reconstruction of $\varphi_{i+1 / 2}^{\prime}$ from $\varphi_{i+1 / 2}$.

Repeating the above procedure, this time with three quadratic interpolants

$$
\begin{aligned}
\tilde{\varphi}_{-}^{[2]}(x)= & \varphi_{i+\frac{1}{2}}+\frac{1}{h}\left(\Delta^{-} \varphi_{i+\frac{1}{2}}\right)\left(x-x_{i+\frac{1}{2}}\right) \\
& +\frac{1}{2 h^{2}}\left(\Delta^{+} \Delta^{-} \varphi_{i+\frac{1}{2}}\right)\left(x-x_{i+\frac{1}{2}}\right)\left(x-x_{i+\frac{3}{2}}\right)+O\left(h^{3}\right), \\
\tilde{\varphi}_{0}^{[2]}(x)= & \varphi_{i+\frac{1}{2}}+\frac{1}{2 h}\left(\Delta^{0} \varphi_{i+\frac{1}{2}}\right)\left(x-x_{i+\frac{1}{2}}\right) \\
& +\frac{1}{2 h^{2}}\left(\Delta^{+} \Delta^{-} \varphi_{i+\frac{1}{2}}\right)\left(x-x_{i-\frac{1}{2}}\right)\left(x-x_{i+\frac{3}{2}}\right)+O\left(h^{3}\right), \\
\tilde{\varphi}_{+}^{[2]}(x)= & \varphi_{i+\frac{1}{2}}+\frac{1}{h}\left(\Delta^{+} \varphi_{i+\frac{1}{2}}\right)\left(x-x_{i+\frac{1}{2}}\right) \\
& +\frac{1}{2 h^{2}}\left(\Delta^{+} \Delta^{+} \varphi_{i+\frac{1}{2}}\right)\left(x-x_{i+\frac{1}{2}}\right)\left(x-x_{i+\frac{3}{2}}\right)+O\left(h^{3}\right),
\end{aligned}
$$

results with

$$
\frac{1}{6} \tilde{\varphi}_{-, i+\frac{1}{2}}^{[2]}+\frac{2}{3} \tilde{\varphi}_{0, i+\frac{1}{2}}^{[[2]}+\frac{1}{6} \tilde{\varphi}_{+, i+\frac{1}{2}}^{\prime[2]}=\varphi_{i+\frac{1}{2}}^{\prime}+O\left(h^{4}\right)
$$

where

$$
\begin{aligned}
& \tilde{\varphi}_{-, i+\frac{1}{2}}^{\prime[2]}=\frac{1}{2 h}\left(\varphi_{i-\frac{3}{2}}-4 \varphi_{i-\frac{1}{2}}+3 \varphi_{i+\frac{1}{2}}\right), \quad \tilde{\varphi}_{0, i+\frac{1}{2}}^{\prime[2]}=\frac{1}{2 h}\left(\varphi_{i+\frac{3}{2}}-\varphi_{i-\frac{1}{2}}\right), \\
& \tilde{\varphi}_{+, i+\frac{1}{2}}^{\prime[2]}=\frac{1}{2 h}\left(-3 \varphi_{i+\frac{1}{2}}+4 \varphi_{i+\frac{3}{2}}-\varphi_{i+\frac{5}{2}}\right) .
\end{aligned}
$$

The fourth-order WENO estimate of $\varphi_{i+1 / 2}^{\prime}$ is

$$
\bar{\varphi}_{i+1 / 2}^{\prime[4]}=w_{i+\frac{1}{2}}^{-} \hat{\varphi}_{-, i+\frac{1}{2}}^{\prime[2]}+w_{i+\frac{1}{2}}^{0}{\varphi^{-\prime 2]}}_{0, \frac{1}{2}}^{[2]}+w_{i+\frac{1}{2}}^{+} \varphi_{+, i+\frac{1}{2}}^{-[2]}
$$

where the weights $w$ are of the form (4) with $c^{-}=c^{+}=1 / 6, c^{0}=2 / 3$, and the oscillatory indicators $S_{i+1 / 2}^{-}=S_{i+1 / 2}[-2,-1], S_{i+1 / 2}^{-}=S_{i+1 / 2}[-1,0]$, and $S_{i+1 / 2}^{+}=S_{i+1 / 2}[0,1]$. 


\subsection{A Fifth-Order Scheme}

Once again, similarly to the third-order scheme, we need to reconstruct the point-values of $\varphi$ and $\varphi^{\prime}$. We start with the reconstruction of $\varphi_{i+1 / 2}$ and $\varphi_{i+1 / 2}^{\prime}$ from $\varphi_{i}$. We write sixth-order interpolants as a convex combination of cubic interpolants, $\varphi_{-}^{[3]}(x)$ and $\varphi_{+}^{[3]}(x)$ introduced above and

$$
\begin{aligned}
\varphi_{0}^{[3]}(x)= & \varphi_{i}+\frac{1}{h}\left(\Delta^{+} \varphi_{i}\right)\left(x-x_{i}\right)+\frac{1}{2 h^{2}}\left(\Delta^{+} \Delta^{-} \varphi_{i}\right)\left(x-x_{i}\right)\left(x-x_{i+1}\right) \\
& +\frac{1}{6 h^{3}}\left(\Delta^{+} \Delta^{-} \Delta^{+} \varphi_{i}\right)\left(x-x_{i}\right)\left(x-x_{i+1}\right)\left(x-x_{i+2}\right)+O\left(h^{4}\right) .
\end{aligned}
$$

In this case

$$
\frac{3}{16} \varphi_{-, i+\frac{1}{2}}^{[3]}+\frac{5}{8} \varphi_{0, i+\frac{1}{2}}^{[3]}+\frac{3}{16} \varphi_{+, i+\frac{1}{2}}^{[3]}=\varphi_{i+\frac{1}{2}}+O\left(h^{6}\right),
$$

where

$$
\begin{aligned}
& \varphi_{-, i+\frac{1}{2}}^{[3]}=\frac{1}{16}\left(\varphi_{i-2}-5 \varphi_{i-1}+15 \varphi_{i}+5 \varphi_{i+1}\right), \\
& \varphi_{0, i+\frac{1}{2}}^{[3]}=\frac{1}{16}\left(-\varphi_{i-1}+9 \varphi_{i}+9 \varphi_{i+1}-\varphi_{i+2}\right), \\
& \varphi_{+, i+\frac{1}{2}}^{[3]}=\frac{1}{16}\left(5 \varphi_{i}+15 \varphi_{i+1}-5 \varphi_{i+2}+\varphi_{i+3}\right) .
\end{aligned}
$$

In a similar way,

$$
-\frac{9}{80} \varphi_{-, i+\frac{1}{2}}^{\prime[3]}+\frac{49}{40} \varphi_{0, i+\frac{1}{2}}^{[3]}-\frac{9}{80} \varphi_{+, i+\frac{1}{2}}^{\prime[3]}=\varphi_{i+1 / 2}^{\prime}+O\left(h^{6}\right),
$$

where

$$
\begin{aligned}
& \varphi_{-, i+\frac{1}{2}}^{[[3]}=\frac{1}{24 h}\left(\varphi_{i-2}-3 \varphi_{i-1}-21 \varphi_{i}+23 \varphi_{i+1}\right), \\
& \varphi_{0, i+\frac{1}{2}}^{[3]}=\frac{1}{24 h}\left(\varphi_{i-1}-27 \varphi_{i}+27 \varphi_{i+1}-\varphi_{i+2}\right), \\
& \varphi_{+, i+\frac{1}{2}}^{[33]}=\frac{1}{24 h}\left(-23 \varphi_{i}+21 \varphi_{i+1}+3 \varphi_{i+2}-\varphi_{i+3}\right) .
\end{aligned}
$$

The sixth-order WENO estimates for $\varphi_{i+1 / 2}$ and $\varphi_{i+1 / 2}^{\prime}$ are

$$
\begin{aligned}
& \varphi_{i+\frac{1}{2}}^{[6]}=w_{i+\frac{1}{2}}^{-} \varphi_{-, i+\frac{1}{2}}^{[3]}+w_{i+\frac{1}{2}}^{0} \varphi_{0, i+\frac{1}{2}}^{[3]}+w_{i+\frac{1}{2}}^{+} \varphi_{+, i+\frac{1}{2}}^{[3]}, \\
& \varphi_{i+\frac{1}{2}}^{\prime[6]}=w_{i+\frac{1}{2}}^{\prime-} \tilde{\varphi}_{-, i+\frac{1}{2}}^{\prime[3]}+w_{i+\frac{1}{2}}^{\prime 0} \tilde{\varphi}_{0, i+\frac{1}{2}}^{\prime[3]}+w_{i+\frac{1}{2}}^{\prime+} \tilde{\varphi}_{+, i+\frac{1}{2}}^{\prime[3]},
\end{aligned}
$$

where the weights for $\varphi$ are given by (4), with $c_{-}=c_{+}=3 / 16, c_{0}=5 / 8$ and the oscillatory indicators are $S_{i+1 / 2}^{-}=S_{i+1 / 2}[-2,0], S_{i+1 / 2}^{0}=S_{i+1 / 2}[-1,1]$ and $S_{i+1 / 2}^{+}=S_{i+1 / 2}[0,2]$. The negative weights for $\varphi^{\prime}$ require special treatment (see [22] for details). Following [22] we split the positive and negative 
weights in the following way: first, we set $\gamma_{-}^{-}=\gamma_{-}^{+}=9 / 40, \gamma_{-}^{0}=49 / 40$ and $\gamma_{+}^{-}=\gamma_{+}^{+}=9 / 80, \gamma_{+}^{0}=49 / 20$. Then, For $k, l \in\{-, 0,+\}$, set $\sigma_{ \pm}=\sum_{k} \gamma_{ \pm}^{k}$ so that similarly to (4),

$$
\alpha_{ \pm, i+\frac{1}{2}}^{k}=\frac{\gamma_{ \pm}^{k}}{\sigma_{ \pm}\left(\epsilon+S_{i+\frac{1}{2}}^{k}\right)^{p}}
$$

and

$$
w_{i+\frac{1}{2}}^{\prime k}=\sigma_{+} \frac{\alpha_{+, i+\frac{1}{2}}^{k}}{\sum_{l} \alpha_{+, i+\frac{1}{2}}^{l}}-\sigma_{-} \frac{\alpha_{-, i+\frac{1}{2}}^{k}}{\sum_{l} \alpha_{-, i+\frac{1}{2}}^{l}} .
$$

Because $\varphi_{i+1 / 2}^{[3]}$ and $\varphi_{i+1 / 2}^{[3]}$ are defined on the same stencils, they use the same smoothness measures $S_{i+1 / 2}$.

All that is left is the reconstruction of $\varphi_{i+1 / 2}^{\prime}$ from $\varphi_{i+1 / 2}$. In this case a sixth-order approximation to $\varphi_{i+1 / 2}^{\prime}$ requires a weighted sum of four cubic interpolants. This reconstruction is similar to the previous ones. We skip the details and summarize the result:

$$
\tilde{\varphi}_{i+\frac{1}{2}}^{\prime[6]}=w_{i+\frac{1}{2}}^{-} \tilde{\varphi}_{-, i+\frac{1}{2}}^{\{3]}+w_{i+\frac{1}{2}}^{0-} \hat{\varphi}_{0-, i+\frac{1}{2}}^{\prime[3]}+w_{i+\frac{1}{2}}^{0+} \tilde{\varphi}_{0+, i+\frac{1}{2}}^{\{[3]}+w_{i+\frac{1}{2}}^{+} \tilde{\varphi}_{+, i+\frac{1}{2}}^{\prime[3]},
$$

where

$$
\begin{aligned}
\tilde{\varphi}_{-, i+\frac{1}{2}}^{\prime[3]} & =\frac{1}{6 h}\left(-2 \varphi_{i-\frac{5}{2}}+9 \varphi_{i-\frac{3}{2}}-18 \varphi_{i-\frac{1}{2}}+11 \varphi_{i+\frac{1}{2}}\right), \\
\tilde{\varphi}_{0-, i+\frac{1}{2}}^{[3]} & =\frac{1}{6 h}\left(\varphi_{i-\frac{3}{2}}-6 \varphi_{i-\frac{1}{2}}+3 \varphi_{i+\frac{1}{2}}+2 \varphi_{i+\frac{3}{2}}\right), \\
\tilde{\varphi}_{0+, i+\frac{1}{2}}^{[3]} & =\frac{1}{6 h}\left(-2 \varphi_{i-\frac{1}{2}}-3 \varphi_{i+\frac{1}{2}}+6 \varphi_{i+\frac{3}{2}}-\varphi_{i+\frac{5}{2}}\right), \\
\tilde{\varphi}_{+, i+\frac{1}{2}}^{[[3]} & =\frac{1}{6 h}\left(-11 \varphi_{i+\frac{1}{2}}+18 \varphi_{i+\frac{3}{2}}-9 \varphi_{i+\frac{5}{2}}+2 \varphi_{i+\frac{7}{2}}\right) .
\end{aligned}
$$

Here, $c_{-}=c_{+}=1 / 20, c_{0}-=c_{0}+=9 / 20, S_{i+1 / 2}^{-}=S_{i+1 / 2}[-3,-1], S_{i+1 / 2}^{0-}=$ $S_{i+1 / 2}[-2,0], S_{i+1 / 2}^{0+}=S_{i+1 / 2}[-1,1]$ and $S_{i+1 / 2}^{+}=S_{i+1 / 2}[0,2]$.

\section{Numerical Examples}

In all our numerical simulations, the ODE solvers we use are the non-linear fourth-order Strong-Stability Preserving Runge-Kutta (SSP-RK) methods of [6].

We start by testing the accuracy of our new CWENO methods when approximating the solution of the linear advection equation, $\varphi_{t}+\varphi_{x}=0$. The initial data is taken as $\varphi(x, 0)=\sin ^{4}(\pi x)$, the mesh ratio $\lambda=0.9$ and the time $T=4$. The results obtained with the fifth-order method of $\S 2.2$ are shown in Table 1 . 
Table 1. Error and convergence rate for linear advection with initial condition $\varphi(x, 0)=\sin ^{4}(\pi x)$

\begin{tabular}{ccc}
\hline $\mathrm{N}$ & $L_{1}$ error & $L_{1}$ order \\
\hline $505.03 \times 10^{-2}$ & - \\
$1008.36 \times 10^{-5}$ & 9.23 \\
$2002.56 \times 10^{-6}$ & 5.03 \\
$4008.24 \times 10^{-8}$ & 4.96 \\
$8002.99 \times 10^{-9}$ & 4.78 \\
\hline
\end{tabular}

Next, we test the CWENO methods with two nonlinear Hamiltonians: a convex Hamiltonian $\varphi_{t}+\frac{1}{2}\left(\varphi_{x}+1\right)^{2}=0$ and a non-convex Hamiltonian $\varphi_{t}-\cos \left(\varphi_{x}+1\right)=0$. The interval is $[0,2]$, the boundary conditions are periodic and the initial conditions for both Hamiltonians are taken as $\varphi(x, 0)=-\cos (\pi x)$. The exact solution to both problems is smooth until $t \approx 1 / \pi^{2}$, after which a singularity forms. A second singularity forms in the non-convex $H$ example at $t \approx 1.29 / \pi^{2}$.

The results of the accuracy test with the fifth-order method are shown in Table 2, and the solution at time $T=1.5 / \pi$ is plotted in Figure 1. Following [9] the errors in Table 2 after the formation of the singularity are computed at a distance of 0.1 away from any singularities.

Table 2. $L_{1}$ Error and convergence rate estimates for convex and non-convex Hamiltonians. top: $T=0.5 / \pi^{2}$, bottom: $T=1.5 / \pi^{2} . \lambda=0.3$

\begin{tabular}{|c|c|c|c|c|}
\hline $\mathrm{N}$ & $\begin{array}{l}\text { convex } \\
L_{1} \text { error }\end{array}$ & $\begin{array}{l}\text { convex } \\
L_{1} \text { order }\end{array}$ & $\begin{array}{c}\text { non-convex } \\
L_{1} \text { error }\end{array}$ & $\begin{array}{c}\text { non-convex } \\
L_{1} \text { order }\end{array}$ \\
\hline $\begin{array}{c}50 \\
100 \\
200 \\
400 \\
800\end{array}$ & $\begin{array}{l}6.35 \times 10^{-6} \\
1.62 \times 10^{-7} \\
5.72 \times 10^{-9} \\
2.73 \times 10^{-10} \\
1.45 \times 10^{-11}\end{array}$ & $\begin{array}{c}- \\
5.30 \\
4.82 \\
4.39 \\
4.23\end{array}$ & $\begin{array}{l}4.17 \times 10^{-5} \\
1.49 \times 10^{-6} \\
4.19 \times 10^{-8} \\
1.34 \times 10^{-8} \\
4.20 \times 10^{-8}\end{array}$ & $\begin{array}{l}- \\
4.81 \\
5.15 \\
4.97 \\
4.99\end{array}$ \\
\hline$N$ & $\begin{array}{l}\text { convex } \\
L_{1} \text { error }\end{array}$ & $\begin{array}{l}\text { convex } \\
L_{1} \text { order }\end{array}$ & $\begin{array}{c}\text { non-convex } \\
L_{1} \text { error }\end{array}$ & $\begin{array}{c}\text { non-convex } \\
L_{1} \text { order }\end{array}$ \\
\hline $\begin{array}{c}50 \\
100 \\
200 \\
400 \\
800\end{array}$ & $\begin{array}{c}2.12 \times 10^{-4} \\
1.03 \times 10^{-5} \\
9.68 \times 10^{-8} \\
6.20 \times 10^{-10} \\
1.90 \times 10^{-11}\end{array}$ & $\begin{array}{l}- \\
4.37 \\
6.73 \\
7.29 \\
5.03\end{array}$ & $\begin{array}{l}2.56 \times 10^{-5} \\
7.80 \times 10^{-7} \\
1.70 \times 10^{-8} \\
5.02 \times 10^{-10} \\
1.71 \times 10^{-11}\end{array}$ & $\begin{array}{c}- \\
5.03 \\
5.52 \\
5.08 \\
4.88\end{array}$ \\
\hline
\end{tabular}



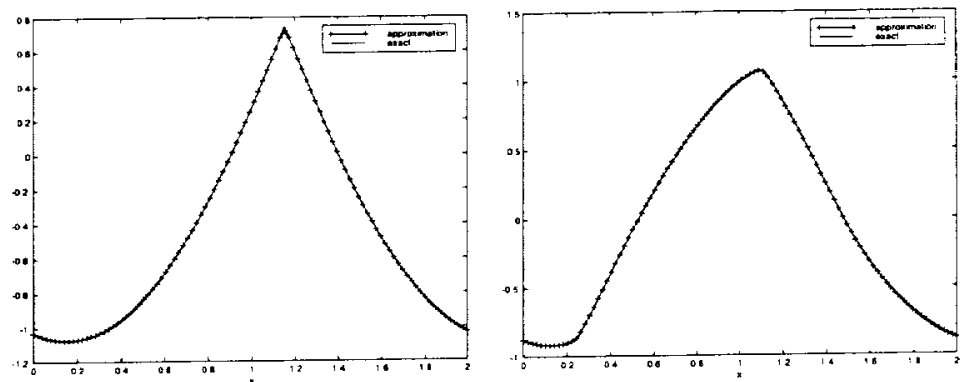

Fig. 1. left: Convex Hamiltonian right: non-convex Hamiltonian at $T=\frac{1.5}{\pi^{2}} \mathrm{com}-$ pared with the exact solution, $N=100$.

\section{References}

1. Barles G., Solution de viscosité des équations de Hamilton-Jacabi, SpringerVerlag, Berlin, 1994.

2. Bryson S., Levy D., Central Schemes for Multi-Dimensional Hamilton-Jacobi Equations, NASA Technical Report NAS-01-012, 2001, submitted.

3. Crandall M.G., Evans L.C., Lions P.-L., Some properties of viscosity solutions of Hamilton-Jacobi equations, Trans. Amer. Math. Soc., 282, (1984), pp.487-502.

4. Crandall M.G., Ishii H., Lions P.-L., User's guide to viscosity solutions of second order partial differential equations, Bull. Amer. Math. Soc., 27, (1992), pp.1-67.

5. Crandall M.G., Lions P.-L., Viscosity solutions of Hamilton-Jacobi equations, Trans. Amer. Math. Soc., 277 (1983), pp.1-42.

6. Gottlieb S., Shu C.-W., Tadmor E., Strong stability-preserving high order time discretization methods, SIAM Review, 43, (2001), pp.89-112.

7. Harten A., Engquist B., Osher S., Chakravarthy S., Uniformly High Order Accurate Essentially Non-oscillatory Schemes III, JCP, 71, (1987), pp.231-303.

8. Kruzkov S.N., The Cauchy problem in the large for nonlinear equations and for certain quasilinear systems of the first order with several variables, Soviet Math. Dokl., 5, (1964), pp.493-496.

9. Jiang G.-S., Peng D., Weighted ENO schemes for Hamilton-Jacobi equations, SIAM J. Sci. Comp., 21, (2000), pp.2126-2143.

10. Jiang G.-S., Shu C.-W., Efficient Implementation of Weighted ENO Schemes, JCP, 126, (1996), pp.202-228.

11. Kurganov A., Noeile S., Petrova G., Semi-discrete central-upwind schemes for hyperbolic conservation laws and Hamilton-Jacobi equations, SIAM J. Sci. Comp., to appear.

12. Kurganov A., Tadmor E., New high-resolution semi-discrete central schemes for Hamilton-Jacobi equations, JCP, 160, (2000), pp.720-724.

13. Levy D., Puppo G., Russo G., Central WENO schemes for hyperbolic systems of conservation laws, Math. Model. and Numer. Anal., 33, no. 3 (1999), pp.547571 .

14. Levy D., Puppo G., Russo G., Compact central WENO schemes for multidimensional conservation laws, SIAM J. Sci. Comp., 22, (2000), pp.656-672.

15. Lions P.L., Generalized solutions of Hamilton-Jacobi equations, Pitman, London, 1982. 
16. Lin C.-T., Tadmor E., $L^{1}$-stability and error estimates for approximate Hamilton-Jacobi solutions, Numer. Math., 87, (2001), pp.701-735.

17. Lin C.-T., Tadmor E., High-resolution non-oscillatory central schemes for approximate Hamilton-Jacobi equations, SIAM J. Sci. Comp., 21, no. 6, (2000), pp. $2163-2186$.

18. Liu X.-D., Osher S., Chan T., Weighted Essentially Non-oscillatory Schemes, JCP, 115, (1994), pp.200-212.

19. Nessyahu H., Tadmor E., Non-oscillatory central differencing for hyperbolic conservation laws, JCP, 87, no. 2 (1990), pp.408-463.

20. Osher S., Sethian J., Fronts propagating with curvature dependent speed: algorithms based on Hamilton-Jacobi formulations, JCP, 79, (1988), pp.12-49.

21. Osher S., Shu C.-W., High-order essentially nonoscillatory schemes for Hamilton-Jacobi equations, SIAM J. Numer. Anal., 28, (1991), pp.907-922.

22. Shi J., Hu C., Shu C.-W., A technique of treating negative weights in WENO schemes, JCP, to appear. 